\title{
Optimal DG Allocation and Sizing for Mitigating Voltage Sag in Distribution Systems Using Particle Swarm Optimization
}

\author{
Sneha patel, A.K. Sharma, PG Scholer, \\ deptt.of electrical engineering, JEC JABALPU R Professor, deptt. Of Electrical Engineering, JEC college \\ Jabalpur
}

\begin{abstract}
The necessity for clean electric power without any power quality problems has persuaded distribution companies to overcome these issues. Sag/swell, flicker and harmonics are these ones. Among them, voltage sag is one of the most important problems. This phenomenon imposes a bad effect on domestic and industrial loads, especially on sensitive ones. An alternative is use of distributed generation (DG) because of its positive impacts such as voltage support, improved reliability, small size and losses reduction, together with improving voltage sag. An approach is proposed based on Particle Swarm Optimization (PSO), capable to establish the optimal DG allocation and sizing on a distribution network. The objective function is the summation of cost of injecting energy of DGs, fixed installation cost of DGs and number of percentage of buses Experiencing voltage sag in a vulnerable zone that multi plied by number of faults for every kind of faults. The results show that the appropriate placement of DG results in improvement of the aforementioned indices.
\end{abstract}

Keywords: - Distribution Networks, PSO Algorithm, Voltage Sag, Optimal DG Placement and Sizing

\section{INTRODUCTION}

Some studies show that within the two or three years, distributed generation may represent up to $30 \%$ of all new generation [1]. Moreover, renewable energy based DGs such as wind or solar can produce nearly zero pollutant Emissions. DGs can operate as backup in case of interruptions, or for peak shaving during high demand [2]-[3]-[4]. The relationship between power quality and distribution system has been a subject of interest for several years. Voltage sags are considered as the most serious problem of power quality. At the same time, distributed Generations from renewable resources are more and more widely used owing to the growing concern on environmental protection. In general, these generation units are connected to the utility grid by a power electronic interface, which has large potential to be utilized to incorporate the function of voltage sag mitigation. Characteristics of voltage sag in distribution networks caused by faults are highly influenced by the placement of DGs and size of them, although generators embedded in a distribution network can help to maintain during fault voltages at the healthy-phase [5],[6]. There are many references about voltage sags and the good effect of distributed generation on them. Collins presented the dynamic behaviour of a synchronous distributed generator connected to a distribution system during fault conditions. A fault on an actual system was found to have sag that was extended after the fault was cleared. It is shown that the combination of the generator and the load on the system contributes to the extended sag. The detailed simulation studies demonstrate that the primary factors are the reactive power drawn by the load during the fault and recovery along with the reaction of the generator. The prediction of such behaviour requires proper modelling of the generator, the generator's controls, and the system and dynamic load.

Operators of such generators need to be aware of this type of system and load response when synchronous DG is connected to a distribution network [7]. Cheng presented the influence of dispersed generation systems (DGs) on the voltage sags in distribution systems. One radial and one closed-loop distribution feeders are selected for study. The DGs with the synchronous and induction generators are assumed to be installed at different sites of the feeders to find the impact on the voltage sags. By integrating the ITIC curve and the SEMI F47 curve into a wider range of ride-through capability curve, and another CBEMA curve have both been adopted in this paper to evaluate the effect of voltage sags. Also, number of voltage sags occurred per year is predicted and presented by performance contour charts for the radial and closed-loop feeders with and without considering the installation of DGs [8]. Martinez discussed about the characteristics of voltage sags caused by faults in distribution networks that depends on the design of the protection system and the coordination between the different protective devices. The presence of distributed generation changes the radial nature of distribution systems, so it can affect the performance of the protection system, and consequently the characteristics of voltage sags. In addition, DG will help to maintain the during-fault voltages of healthy phases. This paper explored the impact that DG can have on voltage sags characteristics by means of a small test distribution network and presents a simple method to compare and rank the performance of protection systems [9]. Wang proposed two configurations of interconnect distributed generator systems to mitigate voltage sags. 
The discrete vector control algorithm which incorporates both inner loop current controller and outer loop voltage controller is derived to control the compensation for both balanced and unbalanced dips. Simulation results show that Controller possesses good dynamic and static performances and the load voltage can be compensated to the reference level. [10].

Jerome developed a new procedure based on a Genetic Algorithm (GA), capable to establish the optimal distributed generation allocation on a MV distribution network, considering the vulnerability of the system to voltage sag. To do so, demonstrate the effect of DG allocation in a network, three indices have been examined. Average RMS Frequency Index, SARFIx; which represents the average number of specified RMS variation events that occurs over the assessment period per customer served the overall sag performance which is the number or percentage of buses experiencing voltage sag and the overall voltage drop which is basically a summation of all voltage drops in the distribution network under study. The first index reveals the effect of the positioning, on the end users, while the other two reflect the effect on the whole network. The most appropriate places for DG installation have been found to be the weakest parts of the network. The results show that the appropriate placement of DG results in a tremendous improvement of the Aforementioned indices [11].

Salha proposed an over current limitation and voltage control strategy for a grid connected inverter with a LC output filter used in distributed generation and in case of voltage sag. This strategy relies on the control of LC output filter voltage with a resonant controller. This controller has to control current and load voltage throughout voltage sag. Generally, resonant controller is made up of a proportional and resonance term, which contains two imaginary poles that aimed to obtain an infinite gain at the resonance frequency. State feedback structure and pole assignment approach are used to tune the proposed control strategy. Comparative results for the application of both resonant and classical PI controller in d-q frame are showed in this paper. The last part deals with the implementation of control strategies on a real time simulation environment [12].

Zhan proposed a three-stage operation scheme to improve on the system voltage sag ride through capability with the power quality control center (PQCC). This scheme works in two stages.

\section{Stage 1}

Function by maintaining the dc-link voltage at the same level as that before the sag. The PQCC input-side inverter would be protected from overloading during sag mitigation.

\section{Stage 2}

Is designed for compensating more severe sags. The purpose of introducing this stage is to decrease the input side current through reducing the active power supplied by the upstream system. It is shown that this strategy can compensate for a voltage deviation of up to approximately double that under Stage 1 . For even more severe sags that cannot be compensated under Stage 2, the PQCC would reduce the dc-link voltage even further, such that the DG supplies the entire protected loads [13]. Surisunthon presented impacts of distributed generation on voltage sag assessment in distribution systems. Voltage sag assessment is analyzed by using the method of fault positions in order to determine area of vulnerability (AOV), voltage sag frequency and voltage sag index [14].

\section{VOLTAGE SAG}

Most of the power quality problems that experienced by consumers are voltage sags. IEEE Standard 1159-1995 states that voltage sag is a brief decrease in the rms line voltage of 10 to 90 percent of the nominal RMS voltage.

The duration of voltage sag is 0.5 cycle to 1 minute. Voltage sag is not a complete interruption of power; it is a temporary drop of supply voltage made the voltage delivered to the consumer is not at the rated voltage. The European standard 50160 defines voltage sag as a sudden reduction of the supply voltage to a value between $90 \%$ and $10 \%$ of the declared supply voltage, followed by a voltage recovery after a short period of time. The MS IEC 61000 series define voltage sag as a sudden reduction in voltage to a value between $90 \%$ to $10 \%$ of nominal voltage for duration of $10 \mathrm{~ms}$ to 60 second. Risky of voltage sag will depend on the magnitude and duration of it and on the sensitivity of electronic equipments. Voltage sags typically appear when there is an abrupt increase in load such as starting large motor loads. It also appears after symmetrical and asymmetrical faults, motor starting, load switching or transformer energizing. Weather such as lightning, animal contact, contamination of insulators, construction accidents, motor vehicle accidents, falling or contact with tree limbs also contribute in voltage sags. A short circuit fault is a typical cause of voltage sag. Single line to ground faults on the utility system is the most common cause of voltage sags in an industrial plant [15].

Theory of particle swarm optimization (PSO) has been growing rapidly. PSO has been used by many applications of several problems. The algorithm of PSO emulates from behavior of animals societies that don't have any leader in their group or swarm, such as bird flocking and fish schooling. Typically, a flock of animals that have no leaders will find food by random, follow one of the members of the group that has the closest position with a food source (potential solution). The flocks achieve their best condition simultaneously through 
communication among members who already have a better situation. Animal which has a better condition will inform it to its flocks and the others will move simultaneously to that place. This would happen repeatedly until the best conditions or a food source discovered. The process of PSO algorithm in finding optimal values follows the work of this animal society. Particle swarm optimization consists of a swarm of particles, where particle represent a potential solution.

Recently, there are several modifications from original PSO. It modifies to accelerate the achieving of the best conditions. The development will provide new advantages and also the diversity of problems to be resolved. Study on the development of PSO is necessary to do to know how far its development, its advantages and disadvantages and how much use this method to settle a problem. Tutorial and theoretical of PSO has made about what is PSO [1], [2], those describe about what PSO is, simple data tested, and comparison with others evolutionarycomputations.

\section{VARIANT OF PSO}

Exploration is the ability of a search algorithm to explore different region of the search space in order to locate a good optimum. Exploitation, on the other hand, is the ability to concentrate the search around a promising area in order to refine a candidate solution[3]. With their exploration and exploitation, the particle of the swarm fly through hyperspace and have two essential reasoning capabilities: their memory of their own best position - local best (lb) and knowledge of the global or their neighbourhood's best - global best (gb).

Position of the particle is influenced by velocity. Let denote the position of particle in the search space at time step; unless otherwise stated, $\mathrm{t}$ denotes discrete time steps. The position of the particle is changed by adding a velocity, to the current position [1]: with, acceleration coefficient and random vector and. Simple example of PSO, there is a function [3]: where denote as a lower limit and as an upper limit. So, PSO procedure can be described by the following steps: First, Assume that the size of the group of particle is N. It is necessary that the size $\mathrm{N}$ is not too large, but also not too small, so that there are many possible positions toward the best solution or optimal. Second, generate initial population with range and by random order to get the. It is necessary if the overall value of the particle is uniformly in the search area.

After that, the particle and the velocity at iteration are denoted as and thus, these initial particles will be. Vector, () is called a particle or vector coordinates of the particle. (Such as: chromosomes in genetic algorithms). Evaluation of the objective function value for each particle and expressed by then calculate the speed of all particles. All particles move towards the optimal point with a velocity. Initially all of the particle velocity is assumed to be zero. Set iteration At the iteration, find the two important parameters for each particle that is:

\section{A. The best value of (the coordinates of particle at iteration) and declare as:}

With the lowest value of objective function (minimization case), which found a particle at all previous iteration. The best value for all particles which found up to the iteration, with the value function the smallest goal / minimum among all particles for all the previous iterations,

\section{B. Calculate the velocity of particle $j$ at iteration $i$ using the following formula using formula (2):}

Where and, respectively, are learning rates for individual ability (cognitive) and social influence (group), and uniformly random numbers are distributed in the interval 0 and 1 . So the parameters and represent weight of memory (position) of a particle towards memory (position) of the groups (swarm). The value of and is usually 2 , so multiply and ensure that the particles will approach the target about half of the difference

\section{Calculate the position or coordinates of particle at the iteration by:}

Evaluation of the objective function value for each particle and expressed as: The last step, check whether the current solution is convergent. If the positions of all particles leading to an equal value, then this is called convergence. If not convergent then step 4 is repeated by updating iterations, by calculating new values from and. This iteration process continues until all particles convergence the same solution. Usually be determined by the termination criteria (Stopping criterion), for example the amount of the excess solution with a solution now previously been very small.

If the current solution is convergent, then the iteration will stop. We do not know whether the final value is the best value. Below are the stopping criteria conditions for the iteration: First, terminate when a maximum number of iterations, or FEs, has been exceeded. Second, Terminate when an acceptable solution has been found, Third, Terminate when no improvement is observed over a number of iteration. Fourth, terminate when the normalized swarm radius is close to zero. Fifth, terminate when the objective function slope is approximately zero. Although the particle has stopped, we do not know whether the particle will pitch on local optima, local minima, global optima or global optima.

In the original particle swarm optimization, there has also a lack of solution, because it is very easy to move to local optima. In certain circumstances, where a new position of the particle equal to global best and 
local best then the particle will not change its position. If that particle is the global best of the entire swarm then all the other particles will tend to move in the direction of this particle. The end of result is the swarm converging prematurely to a local optimum. If the new position of the particle pretty far from global best and local best then the velocity will changing quickly turned into a great value. This will directly affect the particle's position in the next step. For now the particle will have an updated position of great value, as a result, the particle may be out of bounds the search area.

In analysis, PSO has advantages and disadvantages [4]. Advantages of the basic particle swarm optimization algorithm: PSO is based on the intelligence. It can be applied into both scientific research and engineering use. Then PSO have no overlapping and mutation calculation. The search can be carried out by the speed of the particle. During the development of several generations, only the most optimist particle can transmit information onto the other particles, and the speed of the researching is very fast. After that the calculation in PSO is very simple. Compared with the other developing calculations, it occupies the bigger optimization ability and it can be completed easily. The last one is PSO adopts the real number code, and it is decided directly by the solution. The number of the dimension is equal to the constant of the solution.

On the other hands, disadvantages of the basic particle swarm optimization algorithm are the method easily suffers from the partial optimism, which causes the less exact at the regulation of its speed and the direction. Then the method cannot work out the problems of scattering and optimization and the method cannot work out the problems of non-coordinate system, such as the solution to the energy field and the moving rules of the particles in the energy field.

\section{THE PSO ALGORITHM PROCEDURE}

The particle swarm optimizer (PSO) algorithm is first present by Dr. Kennedy and Dr. Eberhart, and is a random evolution method based on intelligent search of the group birds. It has quick convergence speed and optimal searching ability for solving large-scale optimization problems [14]. The PSO-based approach for solving OPDG problem to minimize the loss takes the following steps:

Step 1: Input line and bus data, and bus voltage limits.

Step 2: Step 2: Calculate the loss using distribution load flow based on backward-forward sweep.

Step 3: Randomly generates an initial population (array) of particles with random positions and velocities on dimensions in the solution space. Set the iteration counter $\mathrm{k}=0$.

Step 4: For each particle if the bus voltage is within the limits, calculate the total loss. Otherwise, that particle is infeasible.

Step 5: For each particle, compare its objective value with the individual best. If the objective value is lower than Pbest, set this value as the current Pbest, and record the corresponding particle position.

Step 6: Choose the particle associated with the minimum individual best Pbest of all particles, and set the value of this Pbest as the current overall best Gbest.

Step 7: Update the velocity and position of particle.

Step 8: If the iteration number reaches the maximum limit, go to Step 9. Otherwise, set iteration index $\mathrm{k}=\mathrm{k}+1$, and go back to Step 4.

Step 9: Print out the optimal solution to the target problem. The best position includes the optimal locations and size of, DG, and the corresponding fitness value representing the minimum total real power loss. 


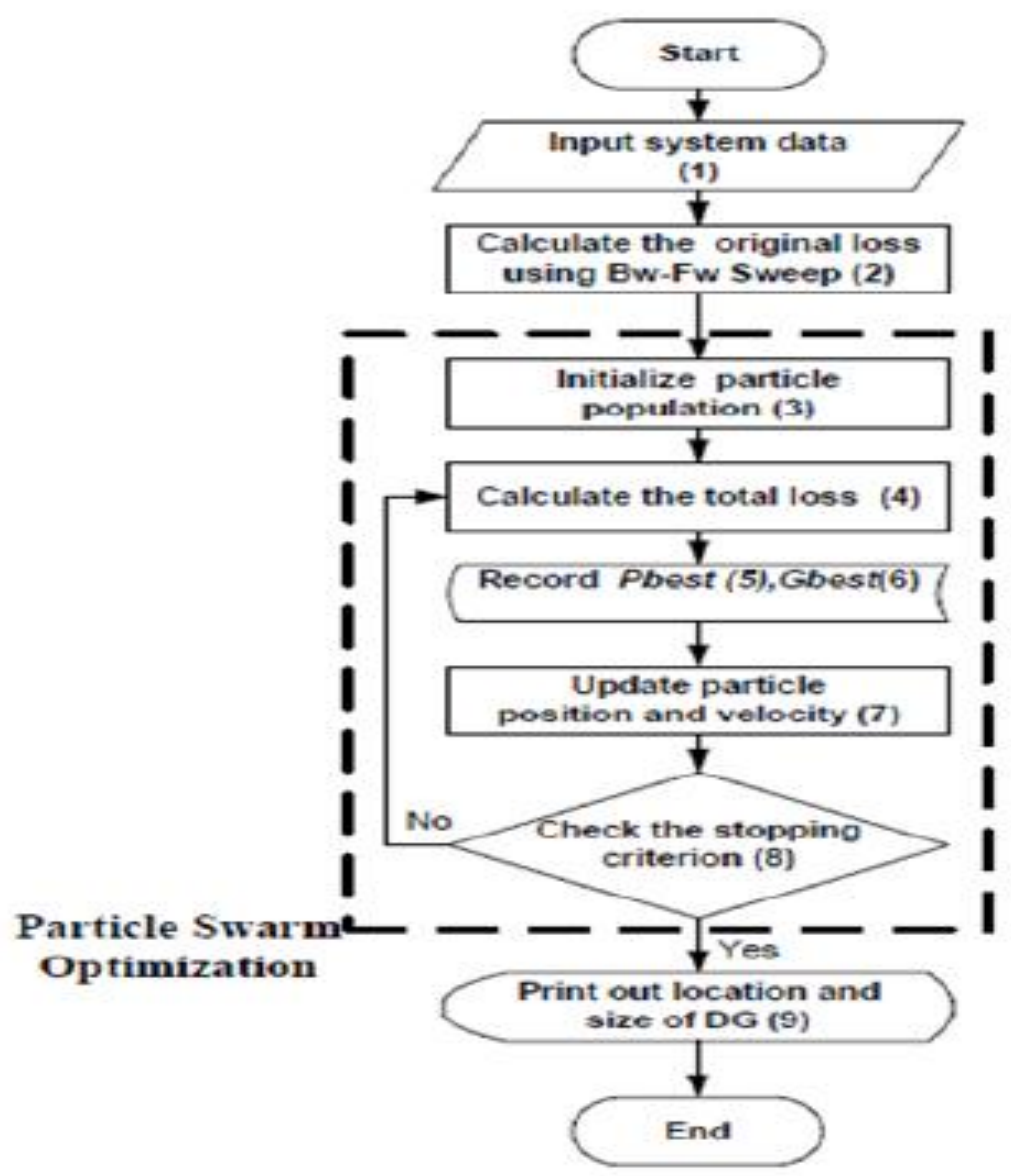

Fig:- (1) PSO computational algorithm Convergence of the PSO algorithm.

The power quality is one of the most booming issues in recent years, largely due to the advance of the electronics and its increasing vulnerability against electrical disturbances. To find the expected value of sags level in the point of common coupling (PCC) on each user, a methodology is proposed consisting in the Following stages:

1) A network modeling, where the more detailed the model, more accurate and suitable are the results for subsequent analysis.

2) An identification of random variables and their trends by analyzing historical available data. Variables such location, fault type and failed phase were represented by means of probability distribution functions that were Stochastically reproduced.

3) A Stochastic simulation of faults, according to the former probability functions by using some techniques such as Gibbs algorithm. This simulation provides information about the expected voltage waveform at each point of the

Feeders.

4) Data analysis. A statistical assessment is performed with the simulations results to determine the sags level (95\% percentile) for each customer. This statistical value establishes the most affected customers.

5) Vulnerable areas identification. The sags level is shown through is-sags maps, which defines contours of equal voltage values when a event occurs. These maps can be used to easily distinguish which areas are more vulnerable

than others.

6) Disturbance areas identification. Through a sensitivity analysis the disturbing areas are identified. Generally, the vulnerable zone is the zone that the consumer experienced voltage sag during the fault. Vulnerability zone of a consumerdepends on the sensitivity to voltage drop. More the sensitivity, the vulnerable zone becomes higher. In order to calculate the rate of voltage sag occurrences that is more than voltage sensitivity of one consumer, it's necessary to calculate its vulnerable zone with respect to different faults. With respect to probability of occurrence of each kind of fault in the vulnerable zone, it's possible to calculate the probability of the number of time that consumer experiences voltage sag, in a specific time period. 


\section{A- Load Model}

Different loads have different vulnerable zone. As an example, three-phase motors can continue their operation for a short period during voltage drops in one or two phases. It's obvious that these kind of loads have small vulnerable zone in comparable with three phases loads that are sensitive to the smallest phase voltage

\section{B- Sensitivity of Loads}

Comparing two kinds of loads, one with larger sensitivity, has larger vulnerable zone.

\section{C- Location of Consumers}

In radial distribution networks, the end user experiences voltage sag during the fault that occurs upstream of it. It means as a large vulnerable zone. However, for a load that is located at the first of feeder, occurrence of any faults in its down-stream, do not causes salient voltage sag unless the fault is near it.

\section{D- Kinds of Faults}

In order to identify the effect of different faults on the vulnerability zone of a consumer, it is sufficient to consider a three-phase load that is sensitive to average voltage of three phases, during occurrence of single phase and three phase faults. If three phases will occurs, each phase drops equally. If one phase voltage will become less than sensitivity, average of voltage become less than sensitivity, too. If single phase fault occurs, the phases occurring the fault will decrease and other phase's voltage do not decrease as much. With respect to these explanations, it is obvious that the vulnerable zone is larger for three phases fault.

\section{CASE STUDY}

test system is a distribution network with 30 buses and PQ loads as shown at figure (2)

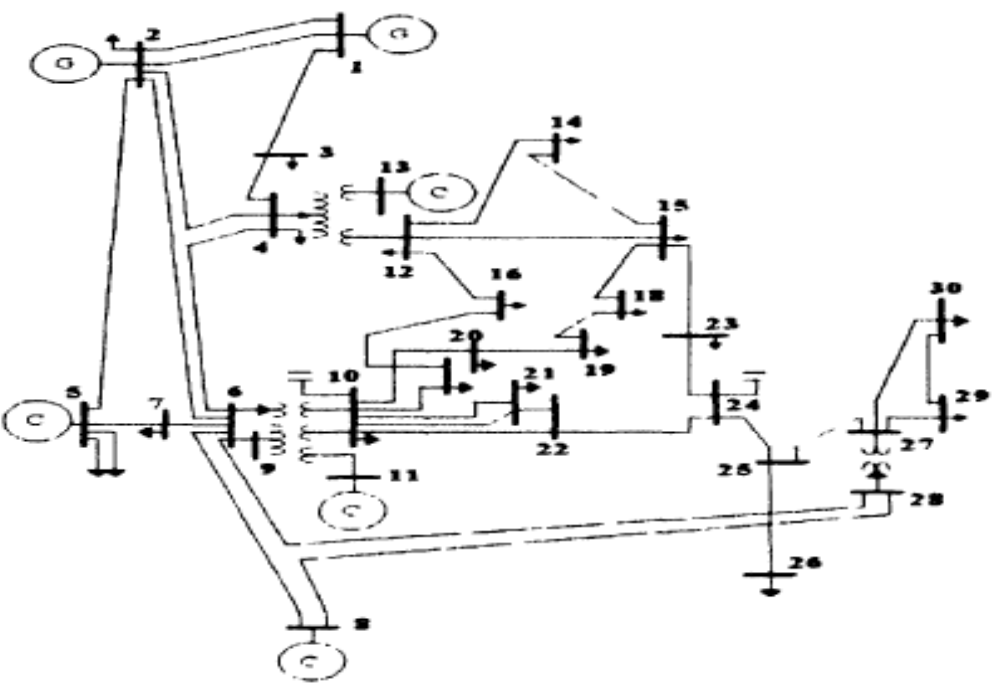

Fig:-(2) The one line diagram of an IEEE-30 bus system

\section{RESULT}

IEEE 33 bus test system is chosen as a study system. Programming is done by "MATLAB". In the PSO algorithm, $\mathrm{n}$ particles for a population are generated randomly where $\mathrm{n}$ is selected to be 40 The goal of the optimization is to find the best location of DGs where the optimization is made on three parameters: its number, location and size. Therefore each particle is a d-dimensional vector in which $d=3$. The number of iteration is considered to be 500, which is the stopping criteria. The parameter in (5) must be tuned. These parameters control the impact of the previous velocities on the current velocity where, in this paper, $\mathrm{c} 1=\mathrm{c} 2=2.05$, the weight $\mathrm{w}$ is decreasing linearly from 0.95 to 0.2 and the turbulence factor is set to be 0.05 . The initialization is made on the position randomly for each particle where each particle shows a number, a location and a size for DGs. Hence the objective functions in this particular case do not conflict each other, archive members is obtained one member, because locating more DG units causes more loss reduction and also less voltage deviation. the candidate location for DG placement are taken from single DG placement algorithm i.e. 6,15, 18, 25, 30, $31,32$. But the number, location, and size of DGs due to global solution are obtained by using MOPSO Algorithm described in section 2. The size of DGs is dependent on the number of DGs locations. Generally it is not possible to install many DGs in a given radial system. In this study the maximum number for DG units has been considered 4 units. Best solution obtained with 4 numbers of DGs. 

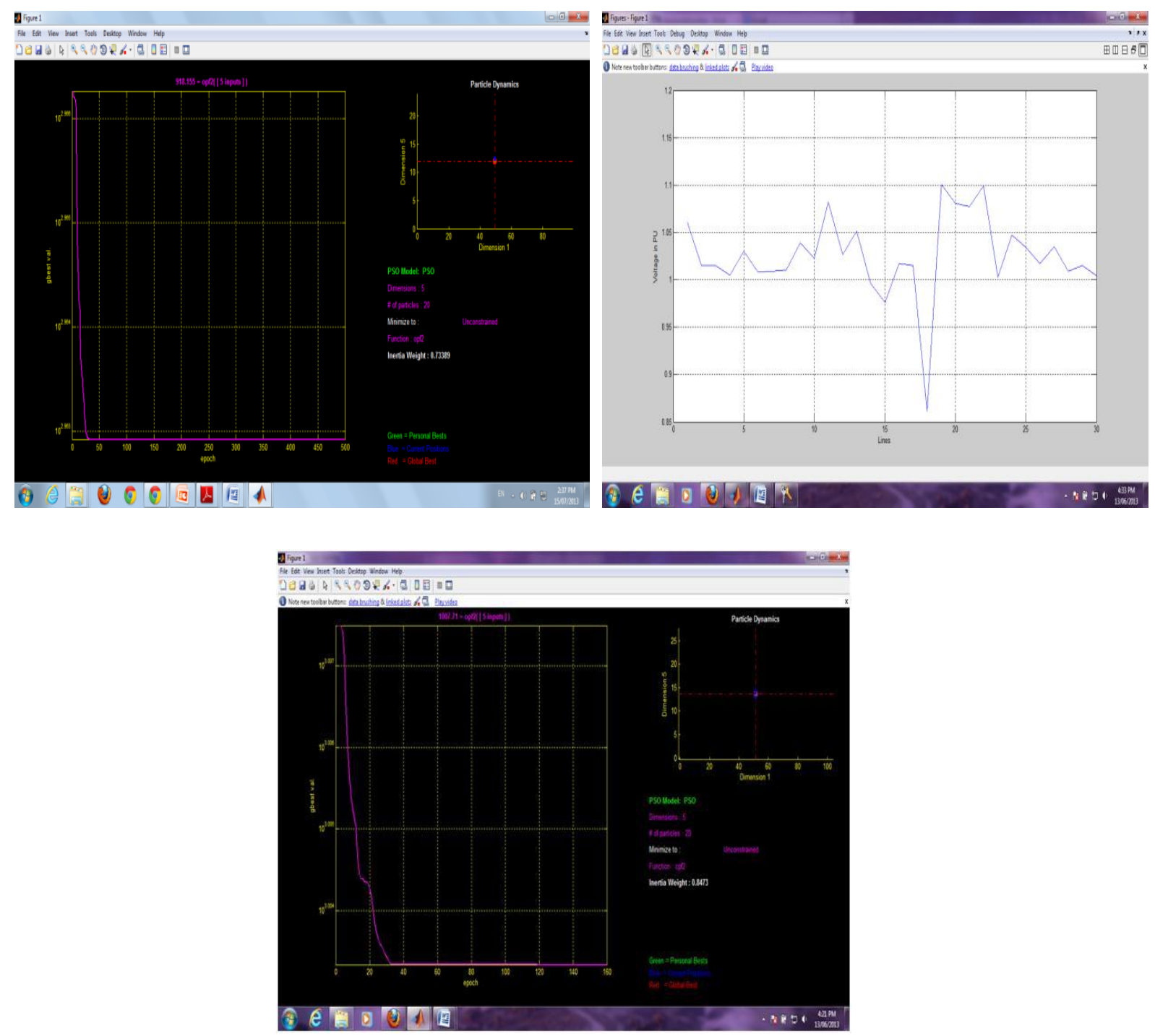

VII. CONCLUSION

In this paper, PSO is applied to determine optimal number, allocation and size of DGs to minimize the voltage sag and reduce the voltage deviation. To find the best local guide the global best method [10] is used. This method is tested on IEEE 30 bus system. By installing DGs, the total power loss of the system has been reduced as well as the system's voltage profile improvement. PSO could be a good alternative for solving such problems specially if the objective functions conflict each other so considering the installation and maintenance cost of DGs could be the future scope of this study.

\section{REFERENCES}

[1]. Lalitha M. Padma, Reddy V. C. Veera,Usha V., Reddy N. Sivarami, Application Of Fuzzy and PSO for DG Placement for Minimum Loss in Radial Distribution System, ARPN Journal of Engineering and Applied Sciences 2010, Vol. 5, No. 4, pp. 30-37

[2]. Walid El-Khattam, Hegazy Y. G.,Salama M. M. A., Investigating Distributed Generation Systems Performance Using Monte Carlo Simulation, IEEE Transactions On Power Systems 2006, Vol. 21, No. 2, pp. 524-532.

[3]. Mithulananthan N., Oo Than, Phu Le Van, Distributed Generation Placement in Power Distribution System Using Genetic Algorithm to Reduce Losses, Thammasat Int. Journal of Science and Technology, Vol.9, No. 3, pp. 55-62.

[4]. Hajizadeh Amin, Hajizadeh Ehsan, PSOBased Planning of Distribution Systems with Distributed Generations, International Journal of Electrical, Computer, and Systems Engineering, 2008, pp. 33-38. 
[5]. Shayeghi H., Bagheri A., Considering DG in Expansion Planning of Subtransmission System, International Conference on Advanced Science, Engineering and Information Technology 2011, pp. 357362.

[6]. Gnativ, R, Milanovic, J.V. " Qualitative and quantitative analysis of voltage sag in network with significant penetration of embedded generation," IEEE Trans on Electrical Power Eng., Vol.24, No. 1, pp. 1734-1741, July 2010.

[7]. Collins, Jian jiang," Voltage sags and the response of a synchronous distributed generator: a case study," IEEE Trans on power del., Vol. 23, No. 1, pp. 442- 448, june 2008.

[8]. Cheng-ting hsu, Chun-jen fu," Dispersed generation systems impact on the voltage sags in distribution systems," international conference on power system technology, pp $1-7$, February 2007.

[9]. Martinez-Velasco, J.A., Martin-Arneclo, J," Distributed generation impact on voltage sags in distribution networks ", 9th International Conference on Electrical Power Quality and Utilization, pp. 1 - 6, January 2008.

[10]. Wang Song-cen, Yu Kun-shan, Tang Guang-fu," Mitigation of voltage sags by grid-connected distributed generation system in series and shunt configurations", IEEE Power Conference on Power System Technology, pp. 1 - 6, January 2009.

[11]. K. T. Chaturvedi, et al., "Particle swarm optimization with time varying acceleration coefficients for nonconvex economic power dispatch," International Journal of Electrical Power \& Energy Systems, vol. 31, pp. 249-257, 2009.

[12]. P. Boonyaritdachochai, et al., "Optimal congestion management in an electricity market using particle swarm optimization with time-varying acceleration coefficients," Computers \& Mathematics with Applications, vol. In Press, Corrected Proof, 2010.

[13]. A. Engelbrecht, "particle Swarm Optimization : Pitfalls and convergen aspect."

[14]. V. Kalivarapu, et al., "Synchronous parallelization of Particle Swarm Optimization with digital pheromones," Advances in Engineering Software, vol. 40, pp. 975-985, 2009.

[15]. S. B. Akat and V. Gazi, "Decentralized asynchronous particle swarm optimization," in Swarm Intelligence Symposium, 2008. SIS 2008. IEEE, 2008, pp. 1-8.

[16]. V. Gazi, "Asynchronous Particle Swarm Optimization," in Signal Processing and Communications Applications, 2007. SIU 2007. IEEE 15th, 2007, pp. 1-4.

[17]. I. Scriven, et al., "Asynchronous multiple objective particle swarm optimisation in unreliable distributed environments," in Evolutionary Computation, 2008. CEC 2008. (IEEE World Congress on Computational Intelligence). IEEE Congress on, 2008, pp. 2481-2486.

[18]. W. Bo, et al., "Distributed Rate Allocation and Performance Optimization for Video Communication Over Mesh Networks," in Image Processing, 2007. ICIP 2007. IEEE International Conference on, 2007, pp. VI - 501-VI - 504.

[19]. Q. Liguo, et al., "Design and Implementation of Intelligent PID Controller Based on FPGA," in Natural Computation, 2008. ICNC '08. Fourth International Conference on, 2008, pp. 511-515.

[20]. T. Desell, et al., "Robust Asynchronous Optimization for Volunteer Computing Grids," in e-Science, 2009. e-Science '09. Fifth IEEE International Conference on, 2009, pp. 263-270.

[21]. L. T. Bui, et al., "A Modified Strategy for the Constriction Factor in Particle Swarm Optimization," in Book Series Lecture Notes in Computer Science vol. Volume 4828/2010, ed. Heidelberg: Springer Berlin, 2010, pp. 333-344.

[22]. L. Bo, et al., "An Effective PSO-Based Memetic Algorithm for Flow Shop Scheduling," Systems, Man, and Cybernetics, Part B: Cybernetics, IEEE Transactions on, vol. 37, pp. 18-27, 2007.

[23]. L. Zhixiong and W. Shaomei, "Hybrid Particle Swarm Optimization for Permutation Flow Shop Scheduling," in Intelligent Control and Automation, 2006. WCICA 2006. The Sixth World Congress on, 2006, pp. 3245-3249.

[24]. L. Dasheng, et al., "A Multiobjective Memetic Algorithm Based on Particle Swarm Optimization," Systems, Man, and Cybernetics, Part B: Cybernetics, IEEE Transactions on, vol. 37, pp. 42-50, 2007.

[25]. Y. G. Petalas, et al., "Enhanced Learning in Fuzzy Simulation Models Using Memetic Particle Swarm Optimization," in Swarm Intelligence Symposium, 2007. SIS 2007. IEEE, 2007, pp. 16-22.

[26]. O. Schutze, et al., "A Memetic PSO Algorithm for Scalar Optimization Problems," in Swarm Intelligence Symposium, 2007. SIS 2007. IEEE, 2007, pp. 128-134.

[27]. L. Hong-qi and L. Li, "A Novel Hybrid Particle Swarm Optimization Algorithm Combined with Harmony Search for High Dimensional Optimization Problems," in Intelligent Pervasive 